\title{
Metastatic L1 Root Compression presenting as an inguinal pain: A Case Report.
}

\author{
Babak Mirzashahi ${ }^{1}$, Farzad Vosoughi ${ }^{1}$, Saied Besharaty ${ }^{1}$, and Sadegh Hasani satehi ${ }^{1}$ \\ ${ }^{1}$ Tehran University of Medical Sciences
}

March 19, 2021

\begin{abstract}
We report a spinal metastatic patient with an atypical presentation of inguinal pain. He was managed with L1 laminectomy and posterior instrumentation. After the surgery, his pain was significantly relieved. This study underscores the importance of spinal examination in those with a known tumor.
\end{abstract}

\section{Metastatic L1 Root Compression presenting as an inguinal pain: A Case Report.}

Abstract:

We report a spinal metastatic patient with an atypical presentation of inguinal pain. He was managed with L1 laminectomy and posterior instrumentation. After the surgery, his pain was significantly relieved. This study underscores the importance of spinal examination in those with a known tumor.

Key words: spinal metastasis, radiculopathy, Laryngeal cancer, Case report

Introduction:

Spine is the most common destination for tumor metastasis after lung and liver ${ }^{1}$ and spine metastasis are the most frequent spinal tumors ${ }^{2}$. Up to 40 percent of patients with tumor are shown to have spine metastasis $^{3}$. Recognition of spinal involvement in those with cancer is important as the spine involvement is first presenting finding in around 10 percent of patients with cancers ${ }^{2}$. Also, symptomatic spinal cord compression due to spine metastasis can lead to an irreversible spinal cord injury within only a week in around one third of cases ${ }^{4}$. Recently, due to improvement in diagnostic and therapeutic armamentarium, the prevalence of spinal metastasis has increased significantly ${ }^{3,5,6}$. So, the need to appropriately diagnose and manage spinal metastasis is strongly felt.

In the following, we present a case of a metastatic vertebral (L1) involvement referred to our clinic with an atypical presentation of severe inguinal pain. Then, we further delineate the diverse clinical manifestation of metastatic spinal diseases reported in the literature.

\section{Case Report:}

A 64-year-old man, known case of Laryngeal cancer undergoing Laryngectomy 12years ago was referred to our clinic with severe Pain at Right inguinal region disabling him for walking. The pain was exacerbating and started from 1 year ago. He had been evaluated by different Specialists. He had taken many analgesics; however, none of them had relieved his chief complaint. His family and social history were insignificant. During physical examination, he had thoracolumbar junction tenderness. Therefore, a lumbosacral plain radiography, lumbosacral Computed Tomography (CT) [figure 1 ] and Magnetic Resonance Imaging (MRI) [figure 2 ] scan was performed. The imaging revealed that he had a L1 vertebral metastasis with accompanying L1 root compression. 
As a result, our patient was managed with L1 vertebral laminectomy and root decompression. The spinal lesion was biopsied and sent for pathology. Posterior instrumentation was performed to enhance the spinal stability [figure 3 ]. Interestingly, the day after surgery inguinal pain was relieved significantly and the patient succeeded to go out of bed with a Thoracolumbosacral orthosis (TLSO). the pathology reported metastatic adenocarcinoma showing papillary configuration with pulmonary origin. Our patient was referred to a Radiotherapist for further radiotherapy treatment. He visited our clinic regularly for follow-up at 2 weeks, 6 weeks, 12 weeks 24 weeks and 1 year postoperatively. After 1 year postoperatively, our patient ambulated independently, his inguinal pain was relieved and had no operative complication.

\section{Discussion:}

The most common complaint in spinal metastatic cases is pain followed by weakness, sensory impairment, bladder or bowel dysfunction ${ }^{2}$. Spinal metastatic cases may have local, mechanical referral or neuropathic pain. Local pain has a deep aching nature, exacerbates while the patient sleeps and improves by NonSteroidal Anti-inflammatory drugs. Mechanical pain is due to the spinal instability. It enhances with changing of position and decrease with lying down. Neuropathic pain is seen in intradural metastasis and has a burning quality. Finally, referral pain is a result of neural root compression caused by the tumor and has a sharp or stabbing nature ${ }^{6}$.

Sciubba et al. stated that only one type of pain may present in spinal metastasis ${ }^{6}$. This is in accordance with our finding, as our patient had a referral pain to the inguinal L1 dermatome without any history suggestive of neuropathic or mechanical pain.

As the spine is the third most common place for metastatic involvement, it is recommended to maintain a high index of suspicion for this diagnosis when managing patients with a known tumor. A back or neck pain in those with a known tumor should be considered spinal metastasis until proven otherwise ${ }^{2}$.

Our patient had no complaint of cervical or back pain; however, on examination, a thoracolumbar tenderness hinted the medical team to evaluate the spinal column. Therefore, this case scenario underscores the importance of spinal examination in those with a known tumor, in order not to miss the spinal metastasis in these patients.

Shaohui He et al. reported the local pain and night aggravating pain as the symptom with most negative and positive predictive value for diagnosing spinal metastasis respectively ${ }^{5}$. Our patient did not complain of a local spinal pain; however, had a local spinal tenderness in the thoracolumbar area.

Symptomatic spinal metastasis most commonly is seen in thoracic spine followed by cervical and lumbar vertebrae $^{2,3}$. In a study performed among patients with breast cancers, the least common spinal metastasis revealed to be thoracolumbar, lumbosacral and sacral vertebrae ${ }^{7}$. In our case, the thoracolumbar area (L1) was involved.

Many studies have demonstrated that symptoms of spinal cord compression caused by spinal metastasis can be effectively improved by spinal decompression surgery. Our finding was similar, as our patient's pain significantly declined immediately after the surgery. Thus, operative management not only may provide spinal metastatic cases with a chance of tumor growth control but also has even more important role in improving the quality of life in these cases.

As a missed spinal metastasis may lead to spinal cord compression, early diagnosis has a paramount importance. This study demonstrates that a spinal metastatic patient needing urgent management may not complain of a neck/ back pain. Given the relative high possibility of spinal metastatic involvement, routine examination of vertebral column in patients with malignancy may help the physician in order not to miss spinal involvement. However, higher level of evidence with larger sample size may give a better understanding of the treatment of spinal metastatic patients.

\section{Conclusion:}


This study highlights the importance of correct history taking and physical examination in managing patients with a known tumor. Complaint of back pain or finding of spinal tenderness should alarm the physician to consider the possibility of spinal metastasis in any patient with a previous history of cancer. Also, Hip pain in any patient should alert the physician to a possibility of upper lumbar root compression.

Authorship list:

B.M conducted the surgery and supervised preparing the manuscript. F.V literature reviewed, and wrote the manuscript. S.B edited the manuscript and collected the patient's data. S.H.S edited the manuscript.

Acknowledgement: none

Funding: None

Conflicts of interest: the authors have completed the ICMJE uniform disclosure form. The authors have no conflicts of interest to declare.

Ethical statement: The authors are accountable for all aspects of the work in ensuring that questions related to the accuracy or integrity of any part of the work are appropriately investigated and resolved. All procedures performed in studies involving human participants were in accordance of ethical standards of the institutional and/or national research committee(s) and with the Helsinki Declaration (as revised in 2013). Written informed consent was obtained from the patient for publication of the Case report and any accompanying images.

\section{References:}

1. Deng Z, Xu B, Jin J, Zhao J, Xu H. Strategies for Management of Spinal Metastases: A Comprehensive Review. Review. Cancer Translational Medicine. May 1, 2015 2015;1(3):94-100. doi:10.4103/23953977.159536

2. Perrin RG, Laxton AW. Metastatic spine disease: epidemiology, pathophysiology, and evaluation of patients. Neurosurg Clin N Am . Oct 2004;15(4):365-73. doi:10.1016/j.nec.2004.04.018

3. Joaquim AF, Powers A, Laufer I, Bilsky MH. An update in the management of spinal metastases. Arq Neuropsiquiatr. Sep 2015;73(9):795-802. doi:10.1590/0004-282x20150099

4. Preciado DA, Sebring LA, Adams GL. Treatment of patients with spinal metastases from head and neck neoplasms. Arch Otolaryngol Head Neck Surg. May 2002;128(5):539-43. doi:10.1001/archotol.128.5.539

5. He S, Ye C, Gao X, et al. Distribution and predictive value of initial presenting symptoms in spinal metastases from primary cancer patients. Eur Spine J . May 6 2020;doi:10.1007/s00586-020-06425-4

6. Sciubba DM, Petteys RJ, Dekutoski MB, et al. Diagnosis and management of metastatic spine disease. A review. J Neurosurg Spine. Jul 2010;13(1):94-108. doi:10.3171/2010.3.spine09202

7. Shehadi JA, Sciubba DM, Suk I, et al. Surgical treatment strategies and outcome in patients with breast cancer metastatic to the spine: a review of 87 patients. Eur Spine J . Aug 2007;16(8):1179-92. doi:10.1007/s00586-007-0357-3

Figure legends:

Fig.1 Preoperative CT scan of our patient. Coronal (A), sagittal (B) and axial (C) reconstructions illustrate a lytic lesion in the body and right sided pedicle of the first lumbar vertebra 
Fig.2 Magnetic Resonance Imaging of the presented patient. It illustrates a spinal lesion which is high signal in T2 (A) and low signal in T1 (B). The axial reconstruction demonstrates an extradural mass compressing the first lumbar nerve root $(\mathrm{C})$

Fig.3 Postoperative plain lumbosacral X ray of the presented patient. Lateral (A) and anteroposterior (B) view is demonstrated

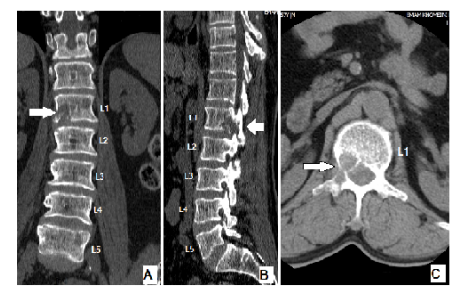

Metastatic L1 Root Compression presenting as an inguinal pain: A Case Report.

Abstract:

We report a spinal metastatic patient with an atypical presentation of inguinal pain. He was

managed with L1 laminectomy and posterior instrumentation. After the surgery, his pain was

significantly relieved. This study underscores the importance of spinal examination in those

with a known tumor.

Key words: spinal metastasis, radiculopathy, Laryngeal cancer, Case report
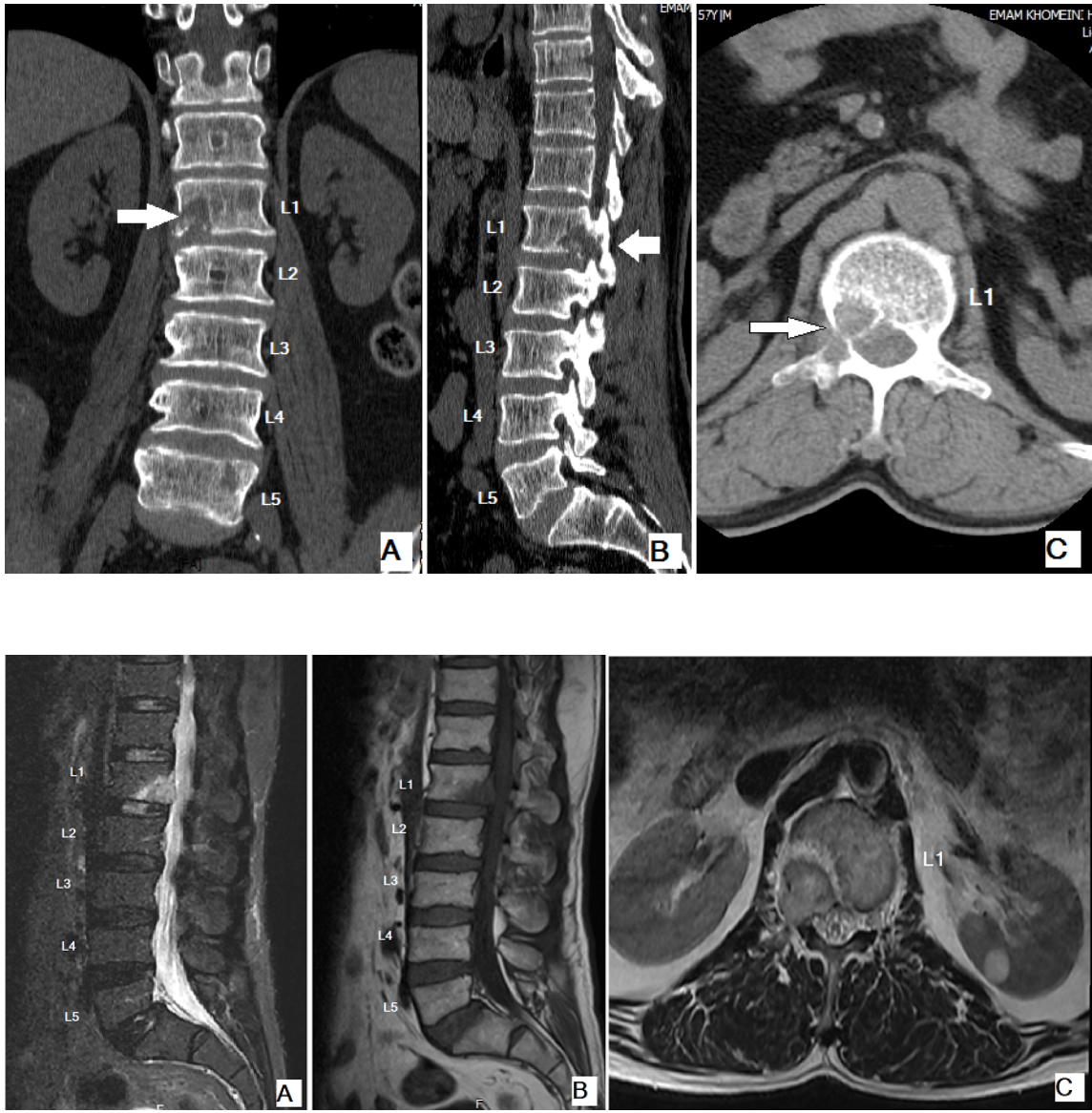


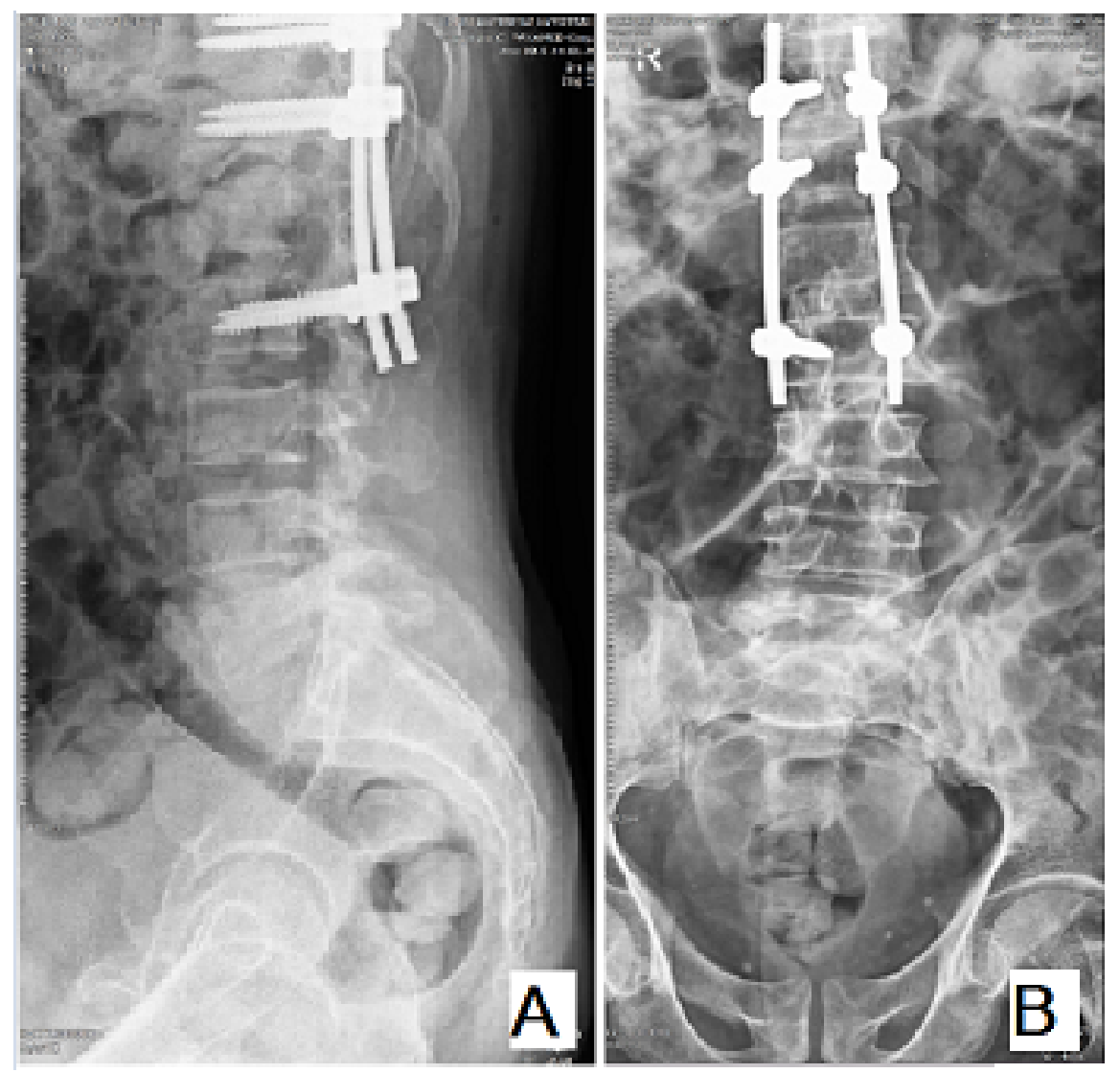

